\title{
Nurses responded on an ad hoc basis to the unpredictable needs of parents of children in hospital
}

\author{
Callery P. Caring for parents of hospitalized children: a hidden area of nursing work. J Adv Nurs 1997 Nov;26:992-8.
}

\section{Question}

What are the perceptions of parents and nurses about the needs of parents who participate in the care of their children in hospital?

\section{Design \\ Qualitative study.}

\section{Setting}

UK.

\section{Participants}

English speaking parents of 24 children who were admitted to a children's hospital for $>24$ hours, had undergone surgery, were not terminally ill, and were under the care of 1 surgeon; and 12 members of the ward staff (10 nurses, 1 health care assistant, and 1 surgeon) who cared for the children in the study.

\section{Methods}

Conversational style interviews with the parents were conducted in the home a few weeks after the children were discharged from hospital. Approximately 125 hours of participant observation were completed during nursing shift handovers, and nursing and medical records were reviewed. Conversational style interviews with the ward staff addressed topics and cases observed during shift handovers as well as topics raised by the participants. Data collection and analysis proceeded concurrently. Interviews were tape recorded and transcribed, and data were analysed using thematic content analysis.

\section{Main results}

Parents experienced distress during involvement in some aspects of their children's care (eg, staying with a child during a painful procedure), but were reluctant to present themselves as needing support. Only after personal support systems were tried and found to be insufficient did parental distress "spill over" and come to the attention of the nurses.

Nurses agreed that they had a responsibility to care for the parents of their patients, but they did not have a common view of the extent or perceived importance of this responsibility. They described their caring interactions with parents as ad hoc responses to unpredictable demands rather than planned interventions. This view was confirmed by observations of discussions during shift handovers.

Nurses felt that parents' demands on their time were unpredictable and led to problems in managing their work. They attributed this unpredictability to uncertainties in the course of the child's illness and care; the process of parents coming to terms with the child's illness; and the personalities of certain parents. Nurses used criteria such as rationality, intelligence, and sensibleness to make judgments about which parents would make demands on their time. In some cases, nurses attributed the difficulties created by demands to "problem parents" rather than to problems in the nurse-parent relationship. For example, anxious behaviour might be labelled as a personality trait or an arbitrary mood of a parent rather than be interpreted as an appropriate response to a specific situation.

\section{Conclusions}

Parents of children in hospital and the nurses caring for their children perceived that (1) parents needed caring from nurses; (2) the nature, extent, and entitlement of such caring was unclear; (3) parents' demands on nurses' time were unpredictable and addressed by nurses on an ad hoc basis; and (4) the unpredictability of demands created time management problems for nurses.

Source of funding: no external funding.

For article reprint:Dr Callery, School of Nursing, Midwifery and Health Visiting, University of Manchester, Coupland III Building, Oxford Road, Manchester M13 9PL, UK. Fax +44 (0)161 2755346.

\section{Commentary}

Although the philosophy of family centred care has been evolving for the past 3 decades, this study by Callery is one of the few that examines the practice of family centred care and explores the implications for nurses. Although the stated purpose of the study was to examine the experiences of parents, Callery, using multiple sources of data, also described the relationships between nurses and parents. The participants were parents and nurses in a surgical nursing unit in the UK, and so the findings may differ for other settings. The work presented in this study helps us to understand the experiences of families and nurses and to recognise the difficulties of implementing family centred care.

Nurses described the struggle they expe- rienced as they attempted to respond to perceived parent needs, which required altering their traditional role. Nursing care was provided to families by exception rather than the norm. Several issues for nurses at all levels of practice were raised. These included recognising parents as partners in the care of their child, that each family is unique, and that a family's needs change over time.

The study findings suggest that nurses and parents need to define their roles and expectations to truly practice family centred care. The findings also reinforce the need to provide learning opportunities and adequate support for nurses to care for families as a whole rather than the child in isolation. The philosophy of fam- ily centred care may need to be more clearly articulated in basic nursing education programmes and continuing education for those working in paediatric settings. If family centred care is clearly articulated as a management expectation, then staffing resources must reflect this commitment. Further research needs to focus on revealing the specific needs of parents and exploring how nurses can plan strategies to enhance the experience of parents and, ultimately, children.

Beth Bruce, RN, MN

Director, Nova Scotia Child Safety and Injury Prevention Program

IWKGrace Health Centre Halifax, Nova Scotia, Canada 